\title{
A Simple Fluorescent Aptasensing Platform Based on Graphene Oxide for Dopamine Determination
}

\section{Ahlem Teniou}

Ecole Nationale Superieure de Biotechnologie de Constantine

amina rhouati ( $\square$ amina.rhouati@gmail.com )

ECOLE NATIONALE SUPERIEURE DE BIOTECHNOLOGIE https://orcid.org/0000-0001-9782-9728

\section{Gaëlle Catanante}

Universite de Perpignan

\section{Research Article}

Keywords: Dopamine, Aptamer, Fluorescence resonance energy transfer, Graphene oxide, Fluorescence quenching

Posted Date: September 24th, 2021

DOl: https://doi.org/10.21203/rs.3.rs-922691/v1

License: @ (i) This work is licensed under a Creative Commons Attribution 4.0 International License. Read Full License

Version of Record: A version of this preprint was published at Applied Biochemistry and Biotechnology on January 8th, 2022. See the published version at https://doi.org/10.1007/s12010-022-03802-1. 


\section{Abstract}

Dopamine (DA) is a catecholamine neurotransmitter playing an important role in different biological functions including central nervous, renal, cardiovascular, and hormonal systems. The sensitive and selective detection of this neurotransmitter plays a key role in the early diagnosis of various diseases related to abnormal levels of dopamine. Therefore, it is of great importance to explore rapid, simple, and accurate methods for detection of dopamine with high sensitivity and specificity. We propose in this work, a fluorescent aptasensor based on graphene oxide (GO) as a quencher, for the rapid determination of dopamine. The principle of this aptasensor is based on fluorescence resonance energy transfer (FRET), where GO was used as energy donor, and a carboxyfluorescein (FAM)-labeled aptamer as acceptor. In the absence of DA, FAM-aptamer was adsorbed on the surface of GO through $\pi-\pi$ stacking interactions between nucleotide bases and the carbon network, leading to a weak FRET and a quenching of the FAM fluorescence. However, by adding the target, the aptamer undergoes a conformational change to bind to DA with high affinity, resulting in a fluorescence recovery. Under the optimal experimental conditions, the fluorescence recovery was linearly proportional to the concentration of DA in the range of 3-1680 nM, with a limit of detection of $0.031 \mathrm{nM}$. Moreover, the developed assay exhibited minor response in the presence of various interferents and it revealed a satisfactory applicability in human serum samples.

\section{Introduction}

Dopamine (DA), is an important neurotransmitter that has a potential vital role in regulating functional activities such as the central nervous, renal, hormonal, and cardiovascular systems [1, 2]. In addition, DA has an important effect on stress, behavior, attention, and other cognitive functions. It has been reported that high dopamine levels induce cardiotoxicity leading to rapid heart rates, hypertension, heart failure, and drug addiction $[3,4]$. In parallel, a low dopamine level may be considered as a major cause of psychiatric disorders such as Parkinson [5], schizophrenia [6], depression [7]...etc [8, 9]. Therefore, the accurate and rapid measurement of DA is crucially required for the diagnosis of these diseases $[10,11]$. Dopamine analysis is usually based on enzyme assays, liquid chromatography, mass spectroscopy, and capillary electrophoresis [12-15]. However, most of these techniques are time-consuming and involve sophisticated pre-treatment process, expensive instruments and expertise for operation thus limiting their applications in routine detection of DA [16-18]. Great efforts are devoted for developing simple, accurate, and inexpensive methods for detecting DA in biological samples with high sensitivity and selectivity.

Among the different technologies, fluorescence resonance energy transfer (FRET) constitutes a promising technique promoting diagnostics. It is a non-radiative energy transfer process that occurs through dipoledipole interaction between an emitter (donor) and an absorber molecule (acceptor) often called the "FRET pair" [19]. FRET can only occur when the intermolecular distance between donors and acceptors is smaller than $10 \mathrm{~nm}$, which allow the detection of interaction between molecules, thus providing accurate information at the nanoscale [20]. This phenomenon is based on the excitation of a donor fluorophore accompanied with an emission spectrum that overlaps the excitation spectrum of an acceptor, in very close proximity $[20,21]$. In this context, fluorescently-labeled aptamers can be used as bioreceptors to 
develop several aptasensors using FRET as a detection method. Based on this principle, the target amount is measured by monitoring the fluorescence change induced by the modification of the aptamer conformation $[22,23]$.

Aptamers are short sequences of DNA or RNA, characterized with high affinity and specificity for their targets [24]. They are selected in vitro by the exponential enrichment process named SELEX (systematic evolution of the ligand by the exponential enrichment process) [25]. As compared to antibodies, aptamers are more stable and economical because they are chemically synthesized [26-28]. In addition, aptamers can be functionalized with different probes including chemical groups [29], enzymes [30] and fluorophores [31]. Aptamer functionalization with a fluorescent probe provides a fundamental advantage in FRET to control the distance between acceptor and donor molecules [32]. Theoretically, any known aptamer can be engineered into a molecular aptamer beacon (MBA) that shows a FRET in response to a specific biomarker [33-35]. Traditional MBA are composed of a fluorophore and a quencher, each attached at the end of hairpin-structured oligonucleotides. The fluorescence is quenched as a result of the proximity between the fluorophore and its quencher. Then, it will be recovered once the optimal distance between the FRET couple is broken [21,36]. However, traditional MBs suffer from some limitations, such as false-positive signals, high-cost synthesis, and difficult selection of dye-quencher pair in certain cases [37]. Therefore, new types of MBs based on nanomaterials, such as gold nanoparticles (AuNPs), quantum dots (QDs), graphene oxide (GO), and carbon nanotubes (CNTs), have been developed as promising candidates for biosensor design [38, 39].

Due to its unique properties, GO has emerged as one of the most extensively studied nanomaterial. It is a two-dimensional (2-D)-layered material synthesized by the oxidation of graphene [40, 41]. GO has excellent electronic, thermal, mechanical and photophysical properties. It has a great potential of applications ranging from molecular electronics to ultrasensitive biosensing [42, 43]. It has been shown that GO has the ability to quench fluorescence of the adsorbed dyes. This is mainly due to the large conjugated structure of GO which makes it an excellent electron acceptor during energy transfer processes [44-46]. These characteristics offer very interesting opportunities to construct a variety of biosensors, in particular, aptasensors [45, 47]. Different GO-based aptasensors has been developed for the detection of different targets such as glucose [48] and pathogens [49]. However, most of the fluorescent methods developed for dopamine aptasensing are mainly based on quantum dots [50,51], nanoclusters[52-54], or nanoparticles [55-57]. The synthesis of such materials involves relatively complicated and time-consuming processes. In this work, we develop a simple fluorescent aptasensor based on graphene oxide as a quencher for DA detection. The principle of this aptasensor is based on FRET by using the FAM-labeled aptamer as a donor and GO as acceptor. First, DA-aptamer was adsorbed onto the surface of $\mathrm{GO}$ where the fluorescence was efficiently quenched. After the target binding, the aptamer changes its conformation in the way that it is released from GO surface, thus recovering the fluorescence that is theoretically proportional to the analyte concentration [58]. This homogeneous fluorescent assay exhibited a high specificity towards DA with a detection limit of $0.031 \mathrm{nM}$. Besides, the application of this sensing system was demonstrated by detecting the DA levels in human serum. Compared with conventional methods, this sensing platform is simpler, faster, and shows 
comprehensible quantitative results. Moreover, it avoids expensive dual labeling of aptamer required in conventional molecular beacon-based platforms.

\section{Material And Methods}

\section{Reagents}

The fluorescein amidite (FAM)-labeled dopamine aptamer with a sequence 5'-FAM-GTC TCT GTG TGC GCC AGA GAA CAC TGG GGC AGA TAT GGG CCA GCA CAG AAT GAG GCC C-3') was synthesized by Microsynth company (Balgach, Switzerland, www.microsynth.ch). Graphene oxide (GO) solution, magnesium chloride $\left(\mathrm{MgCl}_{2}\right)$, sodium chloride $(\mathrm{NaCl})$, hydrochloric acid $(\mathrm{HCl})$, Tris-hydroxymethylmethane (Tris), glycine, glucose, lactic acid and Dopamine (DA) were purchased from Sigma Aldrich (https://www.sigmaaldrich.com). Cysteine was purchased from Biochem (https://www.biochemopharma.fr/). Human serum was obtained from a local biological analysis laboratory. The stock solution of $\mathrm{GO}(4 \mathrm{mg} / \mathrm{ml})$ was used to prepare a homogeneous $\mathrm{GO}$ aqueous solutions of different concentration $(0,2,3,4,5 \mu \mathrm{g} / \mu \mathrm{l})$ using Tris-Hcl buffer $(5 \mathrm{mM} \mathrm{MgCl}, 0.5 \mathrm{M} \mathrm{NaCl}$ and $50 \mathrm{mM}$ Tris $\mathrm{HCl}, \mathrm{pH}$ 7.4), and they were stored at room temperature prior to use. The stock aptamer solution $(2 \mu \mathrm{M})$ was prepared with Tris-HCl buffer $\left(5 \mathrm{mM} \mathrm{MgCl}_{2}, 0.5 \mathrm{M} \mathrm{NaCl}\right.$ and $50 \mathrm{mM}$ Tris HCl, $\left.\mathrm{pH} 7.4\right)$, and stored at $-20^{\circ} \mathrm{C}$ before use. The stock solution of DA $(100 \mu \mathrm{M})$ was also prepared using the same Tris$\mathrm{HCl}$ buffer and stored at $-4^{\circ} \mathrm{C}$. Purified water was used to prepare all the other solutions. All reagents are of analytical grade.

\section{Apparatus}

All fluorescence measurements were recorded with a microplate reader, employing a 96 well black microplate. Aptamer pre-heating was occurred using a thermocycler (Bio-Rad).

\section{Fluorescent detection of dopamine}

The FAM-labeled aptamer $(400 \mathrm{nM})$ was dissolved in Tris-Hcl buffer $\left(5 \mathrm{mM} \mathrm{MgCl}_{2}, 0.5 \mathrm{M} \mathrm{NaCl}\right.$ and 50 $\mathrm{mM}$ Tris $\mathrm{HCl}, \mathrm{pH} 7.4$ ), and then heated to $90^{\circ} \mathrm{C}$ for $5 \mathrm{~min}$. After cooling at room temperature, $25 \mu \mathrm{l}$ of FAMlabeled aptamer was incubated with $25 \mu$ l of different concentrations of DA for 25 min under vortex mixing to make sure that dopamine and DA-aptamer interacted with each other sufficiently. Then $25 \mu l$ of $\mathrm{GO}(3 \mu \mathrm{g} / \mathrm{ml})$ was added to the above solution, and the volume was completed to $200 \mu \mathrm{l}$ using the Tris$\mathrm{Hcl}$ buffer. The mixed solution was allowed to settle under a quit stirring for $30 \mathrm{~min}$ at room temperature. Finally, the fluorescence intensity was measured under the excitation and emission wave- lengths of 485 and $538 \mathrm{~nm}$, respectively. All experiments were repeated three times and carried out under the optimized sensing conditions.

\section{Interference studies}


Aiming to confirm the specificity of the proposed technique for dopamine detection, some interfering compounds (Glycine, cycteine, Glucose, Lactic acid) were tested. The selectivity assay was conducted under the same procedure as for DA detection. In brief, $25 \mu$ of FAM-labeled aptamer (400 nM) dissolved in Tris- $\mathrm{HCl}$ buffer were added to $25 \mu$ of each interferents. Then, the mixture solution was incubated for $25 \mathrm{~min}$ at room temperature, after that $25 \mu \mathrm{l}$ of $\mathrm{GO}(3 \mu \mathrm{g} / \mathrm{ml})$ was introduced to the solution. Finally, the fluorescence was measured after 30 min of quit stirring at room temperature.

\section{Detection of DA in real samples}

In order to confirm the response of the sensor in real samples, human serum samples were diluted 10 times with Tris-Hcl buffer. Then, the samples were spiked with different known concentrations of DA (3,7, $280,1120 \mathrm{nM}$ ). The fluorescence detection of DA in human serum sample was performed under the same procedure mentioned above.

\section{Results And Discussion}

\section{Mechanism of sensing detection}

Figure 1 illustrates the sensing strategy for the DA detection based on the fluorescence resonance energy transfer (FRET) between aptamer and GO. In the absence of target molecule (DA), FAM-modified aptamer is adsorbed onto the surface of $\mathrm{GO}$ via hydrophobic and $\pi-\pi$ stacking interaction between the nucleotide bases and the sp2 honeycomb network of carbon [59-61]. Therefore, these interactions induce the formation of a stable complex that will lead to quench the fluorescence of FAM easily through energy transfer from the FAM to GO [62]. However, in the presence of target molecule (DA), The conformation of DA-aptamer is altered (target-induced allosteric effect), and switched from a random coil to rigid stemloop structure-DA that have a weak affinity to GO and keep the dyes away from graphene surface [63]. Consequently, the FRET process will be hindered, and the FAM fluorescence is recovered and measured as a function of DA. In the experimental mixture, the fluorescence recovery increases by increasing DAconcentrations and the fluorescence will be proportional to the concentration of dopamine $[64,65]$. On the other hand, the addition of interferents couldn't change the conformation of DA-aptamer, so as it can't be released from GO surface, resulting in non-emission of fluorescence due to the quenching effect of GO.

Based on ssDNA-GO interactions, Ye and collaborators [65] demonstrated the generation of a versatile molecular beacon-like probe as a multiplex sensing platform. This probe has been effectively applied as an example of a fluorescent biosensor-based FRET method to detect a specific target, for instance: specific sequence of DNA, as well as thrombin, metal ions such as $\mathrm{Ag}^{+}$and $\mathrm{Hg}^{2+}$, and amino acids such as cysteine, with detection limits of $5 \mathrm{nM}, 20 \mathrm{nM}, 5.7 \mathrm{nM}$, and $60 \mathrm{nM}$, respectively.

\section{Optimization of assay conditions}

Aptamer and quencher concentrations, and incubation time are experimental variables that play an important role in the fabrication of sensitive and selective aptasensing platforms based on FRET 
principle. In this context, different experiments have been realized to optimize aptamer and quencher concentrations, as well as incubation time.

\section{GO concentration}

The choice of GO concentration is a critical factor because it may affect the performance of the sensing system. Optimizations were carried out by varying GO concentration between 0 and $5 \mu \mathrm{g} / \mathrm{ml}$ where the aptamer concentration was fixed at $400 \mathrm{nM}$. The quenching efficiency was defined by $100 \% \times\left(F-F_{0}\right) / F$, where $F_{0}$ and $F$ are the fluorescence intensities of the aptamer solution with and without $G 0$, respectively. As shown in Fig. 2, the quenching of fluorescence increased dramatically with the increasing amounts of $\mathrm{GO}$, indicating that aptamers are still free in the solution. Then, the fluorescence quenching efficiency ( $75 \%$ ) stabilized when the $\mathrm{GO}$ concentration was higher than $3 \mu \mathrm{g} / \mathrm{ml}$, indicating that the FAM-labeled aptamer was almost completely adsorbed onto the surface of GO. Since higher concentrations of GO haven't shown an enhanced quenching efficiency, a concentration of $3 \mu \mathrm{g} / \mathrm{ml}$ was selected as the GO amount generating the optimum quenching. Thus, it has been used in the next experiments.

\section{GO quenching time}

The effect of reaction time between the aptamer and GO on the fluorescence quenching of FAM probe was also investigated. In this context, different incubation times have been tested ranging from 0 to 30 min. As we can see from Fig. 3 , the quenching efficiency increased by increasing the reaction time indicating that the FAM-aptamer was adsorbed onto the surface of GO. After $10 \mathrm{~min}$ of incubation, the signal reached the maximum of $75 \%$ of quenching and stabilized. Therefore, 10 min was chosen to be the optimal incubation time for GO and FAM-aptamer.

\section{Aptamer concentration}

To obtain the maximum sensitivity of biosensing, four different concentrations of aptamer $(200,400,600$ and $800 \mathrm{nM}$ ) were tested. According to the results presented in Fig. 4, the change of fluorescence intensity kept increasing gradually with increasing aptamer concentrations. The optimal concentration is determined according to the sensitivity of the proposed aptasensor. It should be noted that a high concentration of FAM-aptamer provides a better fluorescence signal. However, a high concentration of FAM-aptamer may influence the assay sensitivity leading to an erroneous results [66]. Therefore, the aptamer concentration of $400 \mathrm{nM}$ was adopted to perform the next experiments.

\section{Detection of DA}

The sensitivity of any detection method is considered as a key factor to determine its applicability. It was investigated by monitoring the fluorescence intensity of increasing concentrations of DA at the emission wavelength of $538 \mathrm{~nm}$. The fluorescence intensity was quantified by calculating the percentage of fluorescence recovery $\left[\left(\mathrm{F}-\mathrm{F}_{0}\right) / \mathrm{F}_{0} \times 100\right]$, where $\mathrm{F}$ is the fluorescence intensity in different dopamine concentrations and $F_{0}$ is the fluorescence intensity in the absence of dopamine. The calibration curve 
presenting the fluorescent recovery percentages as function of DA concentrations ranging from $3 \mathrm{nM}$ to $1680 \mathrm{nM}$ was illustrated in Fig. 5. It was revealed that with increasing the concentration of DA, the fluorescence recovery increased gradually with a good linear relationship (Regression coefficient $R^{2}=$ 0.997), indicating the growth of the number of FAM-aptamer attached to the DA target even at low concentrations ( $3 \mathrm{nM}$ ). The detection limit was estimated to be $0.031 \mathrm{nM}$ based on $3 \delta / S$ calculation ( $\delta$ is the standard deviation for the blank solution, and $S$ is the slope of the calibration curve).

The performance of our technique was compared to other electrochemical and optical aptasensors, previously reported in the literature for dopamine detection. It has been noted that the LOD achieved in this new approach is much lower than those of these techniques (Table 1). The high sensitivity of our aptasensor could be attributed to the high affinity of the aptamer toward DA, and the ultra-high fluorescence quenching ability of GO. The change of the conformation of the aptamer upon the addition of target molecules enhanced the distance between FAM-labeled signal probe and the surface of GO, inducing a higher fluorescence restoration. Moreover, since this assay was performed in a homogenous solution, it avoided the need for time-consuming immobilization, coating and washing steps usually required in common heterogeneous assays [67-69]. Indeed, our biosensor provides a simple and rapid detection of DA since it is based on monitoring the fluorescence change due to the target binding. These features, as well as its other merits, such as low cost and fluorescence background, make it a promising tool for a rapid label free detection of DA. 
Table 1

Comparison between this method and other reported techniques for detection of dopamine

\begin{tabular}{|c|c|c|c|c|}
\hline $\begin{array}{l}\text { Detection } \\
\text { method }\end{array}$ & $\begin{array}{l}\text { Detection } \\
\text { range } \\
\mathrm{nM}\end{array}$ & $\begin{array}{l}\text { LOD } \\
\mathrm{nM}\end{array}$ & Principle & Refs \\
\hline \multirow[t]{6}{*}{ Electrochemical } & $\begin{array}{l}5 \times 10^{3}- \\
75 \times 10^{3}\end{array}$ & $\begin{array}{l}3.36 \times \\
10^{3}\end{array}$ & $\begin{array}{l}\text { DNA aptamer/AuNP/rGO/ modified glassy } \\
\text { carbon electrode. }\end{array}$ & [70] \\
\hline & $5-300$ & 2.1 & Au-electrode/Au-NPs/PEI/CNTs. & [67] \\
\hline & $5-150$ & 1 & MB/MCH/DNA/Au electrode. & [71] \\
\hline & $1-1000$ & 0.75 & Aptamer/GCSC-GO/GCE. & [72] \\
\hline & $\begin{array}{l}5 \times 10^{3}- \\
50 \times 10^{3}\end{array}$ & 1000 & $\begin{array}{l}\text { Split aptamer1/split aptamer } 2 \text { / Au- } \\
\text { electrode. }\end{array}$ & [73] \\
\hline & $5-5 \times 10^{2}$ & 1.8 & aptamer/AuNPs/GCE. & [74] \\
\hline \multirow[t]{2}{*}{ Colorimetric } & $540-5400$ & 360 & Aptamer/ unmodified AuNPs. & [75] \\
\hline & $200-1100$ & 70 & AHMT/ AuNPs. & [76] \\
\hline \multirow[t]{6}{*}{ Fluorescence } & $\begin{array}{l}26- \\
2.90 \times 10^{3}\end{array}$ & 2 & DNA/ rhodamine B/ AuNPs. & [68] \\
\hline & $0.1-10.0$ & 0.08 & $\begin{array}{l}\text { tDNA/cDNA/aptamer/dopamine/hDNA/ Exo } \\
\text { III. }\end{array}$ & [69] \\
\hline & $50-2.5 \times 10^{5}$ & 10 & CQDs/AuNPs/aptamer. & [77] \\
\hline & $5 \times 10^{4}-10^{6}$ & $10^{4}$ & $\mathrm{CB} / \mathrm{Fe}^{2+} / \mathrm{DNA}$ & [29] \\
\hline & $30-210$ & 19 & $\mathrm{Ru} / \mathrm{QDs} / \mathrm{DNA}$ & [78] \\
\hline & $3-1680$ & 0.031 & GO/DNA & $\begin{array}{l}\text { This } \\
\text { work }\end{array}$ \\
\hline
\end{tabular}

\section{Selectivity of the sensing system}

Selectivity is another important feature of a good sensing system as though the presence of many interferents in the real sample would affect the accuracy of the detection mechanism. In this regard, the selectivity of the aptamer-GO sensing platform was studied by monitoring the fluorescence recovery percentage for different interferential substances including glycine, cysteine, glucose, and lactic acid. For that, we set the concentration of DA and the other interferents at $1120 \mathrm{nM}$, and then each one was incubated with $25 \mu \mathrm{l}$ of FAM-aptamer. After $25 \mathrm{~min}, 25 \mu \mathrm{l}$ of GO was added to the mixture, and the fluorescence recovery was measured after 30 min of incubation. As shown in Fig. 6, under the same experimental conditions, the fluorescence recoveries of the sensing system in the presence of DA are 
highly significant, whereas a minor fluorescence restoration was observed in the presence of the tested interferents. Furthermore, it can be seen that the fluorescence enhancement in the presence of the target was more than 4-fold as compared to the other interferents. These results indicates that glycine, cysteine, glucose, and acid lactic are not recognized by DA-aptamer. Consequently, the aptamer remains adsorbed on the GO surface inducing the fluorescence quenching. In particular, the aptamer was strongly bound to dopamine, thus inducing its release from the surface of $\mathrm{GO}$ and the fluorescence recovery. Therefore, this confirms that the label-free fluorescent aptasensor based on GO exhibited an excellent specificity for the DA detection, thus indicating its potential application in complex matrices.

\section{Determination of DA in human serum}

Table 2

Recoveries of DA from human serum samples $(n=3)$

\begin{tabular}{|lllll|}
\hline Sample & Standard value of DA $(\mathrm{nM})$ & Found $(\mathrm{nM})$ & Recovery $(\%)$ & RSD $(\%, \mathrm{n}=3)$ \\
\hline Human serum & 3 & 3.02 & 100.53 & 0.76 \\
\cline { 2 - 5 } & 7 & 7.01 & 100.21 & 2.09 \\
& 280 & 288.8 & 103.16 & 0.61 \\
\cline { 2 - 5 } & 1200 & 1999.83 & 99.99 & 1.89 \\
\hline
\end{tabular}

In order to investigate the applicability of the method, our biosensor was tested in human serum samples. For that, the samples free of DA were collected from a local biomedical analysis laboratory. Then, they were diluted 10 times and spiked with 3, 7, 280, and $1200 \mathrm{nM}$ of DA. After incubation with the aptamer, 25 $\mu \mathrm{l}$ of $\mathrm{GO}$ was added and the fluorescence recovery was measured. As it is shown in Fig. 7, the fluorescent recovery percentages of human serum and tris-buffer were almost the same. This latter conclusion is confirmed by the analytical results for the samples spiked with DA presented in Table 2. As illustrated in this table, the developed aptasensor exhibited good recoveries ranging from 89-103\%, with RSD between 0.61 and $2.09 \%$. These experimental results confirm the good reliability and applicability of the proposed method for DA detection in complex biological samples.

\section{Conclusion}

This work describes the first label-free fluorescent aptasensor for DA detection using GO as a quencher. The developed aptasensor showed a linear relationship between the fluorescence recovery and DA concentration in the range of 3-1680 nM. In addition, the present sensing platform exhibited an excellent selectivity and sensitivity. Moreover, the applicability of this sensing platform was confirmed by detecting DA in complex biological matrices, with a prominent accuracy. By comparing the present technique to that previously reported in the literature, our results have shown a high accuracy and reliability for rapid analysis of dopamine. Furthermore, the developed device does not require any surface functionalization, thus simplifying the fabrication process as well as the analysis. Therefore, the ssDNA-GO platform could 
be an excellent alternative to universal molecular beacons in constructing sensing systems. It could be potentially applied in biomedical diagnostics for dopamine monitoring as well as other biomarkers.

\section{Declarations}

Acknowledgements This work was financially supported by Bioengineering laboratory, Higher national school of biotechnology, Constantine-Algeria

Fundings No funding

Availability of data and material (data transparency) Not applicable

Code availability Not applicable

Conflict of interests The authors declare no competing interests.

\section{References}

1. Shang, N. G., Papakonstantinou, P., McMullan, M., Chu, M., Stamboulis, A., Potenza, A. ... Marchetto, H. J. A. (2008). f. m. Catalyst-free efficient growth, orientation and biosensing properties of multilayer graphene nanoflake films with sharp edge planes. 18,3506-3514.

2. De Witte, P. J. A., \& PRESS-, A. L. P. (1996). The role of neurotransmitters in alcohol dependence: animal research. 31,13-16.

3. Kirchon, A., Feng, L., Drake, H. F., Joseph, E. A., \& Zhou, H. C. J. C. S. R. (2018) From fundamentals to applications: a toolbox for robust and multifunctionalMOF materials.47,8611-8638.

4. Bucolo, C., Leggio, G. M., Drago, F., \& Salomone, S. J. P. therapeutics(2019). Dopamine outside the brain: The eye, cardiovascular system and endocrine pancreas. 203, 107392.

5. Napier, T. C., Kirby, A., Persons, A. L., \& Psychiatry, B. J. P. i. N.-P., (2020). The role of dopamine pharmacotherapy and addiction-like behaviors in Parkinson's disease. 102, 109942.

6. Xu, Y., Qiu, Z., Zhu, J., Liu, J., Wu, J., Tao, J., \& Chen, L. J. B. n(2019). The modulation effect of noninvasive brain stimulation on cognitive function in patients with mild cognitive impairment: a systematic review and meta-analysis of randomized controlled trials. 20,1-11.

7. Whitton, A. E., Reinen, J. M., Slifstein, M., Ang, Y. S., McGrath, P. J., losifescu, D. V. ... Schneier, F. R. J. B. (2020). Baseline reward processing and ventrostriatal dopamine function are associated with pramipexole response in depression. 143,701-710.

8. Chen, P. Y., Vittal, R., Nien, P. C., \& Ho, K. C. J. B. Bioelectronics(2009). Enhancing dopamine detection using a glassy carbon electrode modified with MWCNTs, quercetin, and Nafion ${ }^{\circledR}$. 24,3504-3509.

9. Naranjo, C. A., Tremblay, L. K., Busto, U. E., \& Psychiatry, B. J. P. i. N.-P., (2001). The role of the brain reward system in depression. 25, 781-823. 
10. Zhang, A., Neumeyer, J. L., \& Baldessarini, R. J. (2007). Recent Progress in Development of Dopamine Receptor Subtype-Selective Agents: Potential Therapeutics for Neurological and Psychiatric Disorders. Chemical Reviews, 107, 274-302

11. Liu, A., Wei, M. D., Honma, I., \& Zhou, H. (2006). Biosensing Properties of TitanateNanotube Films: Selective Detection of Dopamine in the Presence of Ascorbate and Uric Acid. 16,371-376.

12. Nichkova, M., Wynveen, P. M., Marc, D. T., Huisman, H., \& Kellermann, G. H. (2013). Validation of an ELISA for urinary dopamine: applications in monitoring treatment of dopamine-related disorders. $125,724-735$.

13. Hows, M. E. P., Lacroix, L., Heidbreder, C., Organ, A. J., \& Shah, A. J. (2004). High-performance liquid chromatography/tandem mass spectrometric assay for the simultaneous measurement of dopamine, norepinephrine, 5-hydroxytryptamine and cocaine in biological samples. Journal of Neuroscience Methods, 138, 123-132

14. Qian, T., Yu, C., Zhou, X., Wu, S., \& Shen, J. (2014). Au nanoparticles decorated polypyrrole/reduced graphene oxide hybrid sheets for ultrasensitive dopamine detection. Sensors and Actuators $B$ : Chemical, 193, 759-763

15. Lakard, S., Pavel, I. A., \& Lakard, B. (2021). Electrochemical Biosensing of Dopamine Neurotransmitter. A Review, 11, 179

16. Pérez-Fernández, V., Harman, D. G., Morley, J. W., \& Cameron, M. A. (2017). Optimized method to quantify dopamine turnover in the mammalian retina. J. A. C, 89, 12276-12283

17. Matuszewski, B., Constanzer, M., \& Chavez-Eng, C. J. A. c(2003). Strategies for the assessment of matrix effect in quantitative bioanalytical methods based on HPLC - MS/MS. 75, 3019-3030.

18. Wei, N., Zhao, X. E., Zhu, S., He, Y., Zheng, L., Chen, G. ... Liu, Z. J. T. (2016). Determination of dopamine, serotonin, biosynthesis precursors and metabolites in rat brain microdialysates by ultrasonic-assisted in situ derivatization-dispersive liquid-liquid microextraction coupled with UHPLC-MS/MS. 161,253-264.

19. Sahoo, H. J. J., O., P., \& Reviews, P. C. P. (2011). Förster resonance energy transfer-A spectroscopic nanoruler: Principle and applications. 12, 20-30.

20. Medintz, I. L., \& Hildebrandt, N. (2013). FRET-Förster resonance energy transfer: from theory to applications. John Wiley \& Sons

21. Pehlivan, Z. S., Torabfam, M., Kurt, H., Ow-Yang, C., Hildebrandt, N., \& Yüce, M. J. M. A. (2019). Aptamer and nanomaterial based FRET biosensors: a review on recent advances (2014-2019). $186,1-22$.

22. Didenko, V. V. J. B. (2001). DNA probes using fluorescence resonance energy transfer (FRET): designs and applications. 31, 1106-1121.

23. Shi, J., Tian, F., Lyu, J., \& Yang, M. J. J. o. m. c. B. (2015) Nanoparticle based fluorescence resonance energy transfer (FRET) for biosensing applications. 3, 6989-7005.

24. Jayasena, S. D. (1999). Aptamers: An Emerging Class of Molecules That Rival Antibodies in Diagnostics. Clinical Chemistry, 45, 1628-1650 
25. Ma, X., Wang, W., Chen, X., Xia, Y., Wu, S., Duan, N., \& Wang, Z. J. E. F. R. Technology(2014). Selection, identification, and application of Aflatoxin B1 aptamer. 238,919-925.

26. Shim, W. B., Kim, M. J., Mun, H., \& Kim, M. G. J. B. Bioelectronics(2014). An aptamer-based dipstick assay for the rapid and simple detection of aflatoxin B1. 62,288-294.

27. Seok, Y., Byun, J. Y., Shim, W. B., \& Kim, M. G. J. A. C. A. (2015). A structure-switchable aptasensor for aflatoxin B1 detection based on assembly of an aptamer/split DNAzyme. 886,182-187.

28. Toh, S. Y., Citartan, M., Gopinath, S. C., \& Tang, T. H. J. B. bioelectronics(2015). Aptamers as a replacement for antibodies in enzyme-linked immunosorbent assay. 64, 392-403.

29. Seto, D., Maki, T., Soh, N., Nakano, K., Ishimatsu, R., \& Imato, T. (2012). A simple and selective fluorometric assay for dopamine using a calcein blue-Fe2 + complex fluorophore. Talanta, 94, 3643

30. Salimi, A., Khezrian, S., Hallaj, R., \& Vaziry, A. (2014). Highly sensitive electrochemical aptasensor for immunoglobulin E detection based on sandwich assay using enzyme-linked aptamer. Analytical Biochemistry, 466, 89-97

31. He, Y., Lin, Y., Tang, H., \& Pang, D. J. N. (2012). A graphene oxide-based fluorescent aptasensor for the turn-on detection of epithelial tumor marker mucin 1. 4,2054-2059.

32. Yüce, M., Ullah, N., \& Budak, H. J. A. (2015). Trends in aptamer selection methods and applications. $140,5379-5399$.

33. Yamamoto, R., \& Kumar, P. K. J. G. t. c(2000). Molecular beacon aptamer fluoresces in the presence of Tat protein of HIV-1. 5, 389-396.

34. Fang, X., Li, J. J., Perlette, J., Tan, W., \& Wang, K. (2000). Peer reviewed: molecular beacons: novel fluorescent probes. ACS Publications

35. Vo-Dinh, T. (2003). Novel Fluorescent Molecular Beacon DNA Probes for Biomolecular Recognition (pp. 1533-1556). CRC Press

36. Alivisatos, P. J. N. (2004). The use of nanocrystals in biological detection. 22,47-52.

37. Song, S., Liang, Z., Zhang, J., Wang, L., Li, G., \& Fan, C. J. A. C. (2009). Gold-nanoparticle-based multicolor nanobeacons for sequence-specific. DNA analysis, 121, 8826-8830

38. Wang, H., Li, J., Liu, H., Liu, Q., Mei, Q., Wang, Y. ... Lu, Z. J. N. A. R(2002). Label-free hybridization detection of a single nucleotide mismatch by immobilization of molecular beacons on an agarose film. 30,e61-e61.

39. Mairal, T., Cengiz Özalp, V., Lozano Sánchez, P., Mir, M., Katakis, I., \& O’Sullivan, C. K. (2008). Aptamers: molecular tools for analytical applications. Analytical and Bioanalytical Chemistry, 390, 989-1007

40. Hunt, A., Dikin, D. A., Kurmaev, E. Z., Boyko, T. D., Bazylewski, P., Chang, G. S., \& Moewes, A. (2012). Epoxide Speciation and Functional Group Distribution in Graphene Oxide. Paper-Like Materials, 22, 3950-3957 
41. Gao, W., Alemany, L. B., Ci, L., \& Ajayan, P. M. (2009). New insights into the structure and reduction of graphite oxide. Nature Chemistry, 1, 403-408

42. Lee, J., Kim, J., Kim, S., \& Min, D. H. (2016). J. A. d. d. r. Biosensors based on graphene oxide and its biomedical application, 105, 275-287

43. Wang, C. F., Sun, X. Y., Su, M., Wang, Y. P., \& Lv, Y. K. J. A. (2020). Electrochemical biosensors based on antibody, nucleic acid and enzyme functionalized graphene for the detection of disease-related biomolecules. 145,1550-1562.

44. Dong, H., Gao, W., Yan, F., Ji, H., \& Ju, H. J. A. c(2010). Fluorescence resonance energy transfer between quantum dots and graphene oxide for sensing biomolecules. 82, 5511-5517.

45. Chung, C., Kim, Y. K., Shin, D., Ryoo, S. R., Hong, B. H., \& Min, D. H.J. A. O. C. r. (2013) Biomedical applications of graphene and graphene oxide.46,2211-2224.

46. Yuan, H., Qi, J., Xing, C., An, H., Niu, R., Zhan, Y. ... Wang, B. J., A. F. M.. (2015). Graphene-OxideConjugated Polymer Hybrid Materials for Calmodulin Sensing by Using. FRET Strategy, 25, 44124418

47. Hermann, T., \& Patel, D. J. J. S. (2000). Adaptive recognition by nucleic acid aptamers. $287,820-825$.

48. Liu, Y., Yu, D., Zeng, C., Miao, Z., \& Dai, L. J. L. (2010). Biocompatible graphene oxide-based glucose biosensors. 26,6158-6160.

49. Jung, J. H., Cheon, D. S., Liu, F., Lee, K. B., \& Seo, T. S. (2010). A graphene oxide based immunobiosensor for pathogen detection. J. A. C, 122, 5844-5847

50. Zhao, J., Zhao, L., Lan, C., Zhao, S. J. S., \& Chemical, A. B. (2016). Graphene quantum dots as effective probes for label-free fluorescence detection of dopamine. 223,246-251.

51. Ma, Y., Chen, A., Xie, X., Wang, X., Wang, D., Wang, P. ... Li, Y. J. T. (2019). Doping effect and fluorescence quenching mechanism of $\mathrm{N}$-doped graphene quantum dots in the detection of dopamine. 196,563-571.

52. He, W., Gui, R., Jin, H., Wang, B., Bu, X., \& Fu, Y. J. T. (2018). Ratiometric fluorescence and visual imaging detection of dopamine based on carbon dots/copper nanoclusters dual-emitting nanohybrids. 178,109-115.

53. Govindaraju, S., Ankireddy, S. R., Viswanath, B., Kim, J., \& Yun, K. J. S. r(2017). Fluorescent gold nanoclusters for selective detection of dopamine in cerebrospinal fluid. 7, 1-12.

54. Ling, Y., Wang, L., Zhang, X. Y., Wang, X. H., Zhou, J., Sun, Z. ... Chemical, A. B. (2020). Ratiometric fluorescence detection of dopamine based on effect of ligand on the emission of Ag nanoclusters and aggregation-induced emission enhancement. 310,127858.

55. Sivakumar, P., Priyatharshni, S., \& Kumar, K. J. M. C. Physics(2020). Fluorescent silver nanoparticles for sensitive and selective detection of dopamine. 240,122167.

56. Qi, W., Zhao, M., Fu, Y., He, H., Tian, X., Wu, D. ... Hu, P. P. J. D. Pigments(2020). Fluorescent detection of uric acid through photoinduced electron transfer using luminol-terbium (III) nanoparticles synthesized via aggregation-induced fluorescence strategy. 172,107797. 
57. Lin, J., Huang, B., Dai, Y., Wei, J., \& Chen, Y. J. M. S. C, E(2018). Chiral ZnO nanoparticles for detection of dopamine. 93,739-745.

58. Zhu, Y., Cai, Y., Xu, L., Zheng, L., Wang, L., Qi, B., \& Xu, C. J. A. a. m., interfaces(2015). Building an aptamer/graphene oxide FRET biosensor for one-step detection of bisphenol A. 7, 7492-7496.

59. Zheng, M., Jagota, A., Semke, E. D., Diner, B. A., McLean, R. S., Lustig, S. R., \& Richardson, R. E., Tassi, N. G. J. N. m.. (2003). DNA-assisted dispersion and separation of carbon nanotubes, 2, 338-342

60. Mao, B., Calatayud, D. G., Mirabello, V., Kuganathan, N., Ge, H., Jacobs, R. M. ... Hodges, B. J. J. C. (2017). Fluorescence-Lifetime Imaging and Super-Resolution Microscopies Shed Light on the Directed-and Self-Assembly of Functional Porphyrins onto Carbon Nanotubes and Flat Surfaces. 23,9772.

61. Chang, H., Tang, L., Wang, Y., Jiang, J., \& Li (2010). Graphene fluorescence resonance energy transfer aptasensor for the thrombin detection. J. J. A. C, 82, 2341-2346

62. Duan, Y. F., Ning, Y., Song, Y., \& Deng, L. J. M. A. (2014). Fluorescent aptasensor for the determination of Salmonella typhimurium based on a graphene oxide platform. 181,647-653.

63. Walsh, R., \& DeRosa, M. C. J. B. communications, b. r(2009). Retention of function in the DNA homolog of the RNA dopamine aptamer. 388, 732-735.

64. Yang, M., Javadi, A., Li, H., \& Gong, S. J. B. Bioelectronics(2010). Ultrasensitive immunosensor for the detection of cancer biomarker based on graphene sheet. 26,560-565.

65. Zhang, M., Yin, B. C., Tan, W., \& Ye, B. C. J. B., Bioelectronics. (2011). A versatile graphene-based fluorescence "on/off". switch for multiplex detection of various targets, 26, 3260-3265

66. Goud, K. Y., Sharma, A., Hayat, A., Catanante, G., Gobi, K. V., Gurban, A. M., \& Marty, J. L. (2016). Tetramethyl-6-carboxyrhodamine quenching-based aptasensing platform for aflatoxin B1: Analytical performance comparison of two aptamers. J. A. b, 508, 19-24

67. Azadbakht, A., Roushani, M., Abbasi, A. R., Menati, S., \& Derikvand, Z. (2016). A label-free aptasensor based on polyethyleneimine wrapped carbon nanotubes in situ formed gold nanoparticles as signal probe for highly sensitive detection of dopamine. Materials Science and Engineering: C, 68, 585-593

68. Xu, J., Li, Y., Wang, L., Huang, Y., Liu, D., Sun, R. ... Sun, C. (2015). A facile aptamer-based sensing strategy for dopamine through the fluorescence resonance energy transfer between rhodamine $B$ and gold nanoparticles. Dyes and Pigments, 123, 55-63

69. Wang, Y., Kang, K., Wang, S., Kang, W., Cheng, C., Niu, L. M., \& Guo, Z. (2020). A novel label-free fluorescence aptasensor for dopamine detection based on an Exonuclease III- and SYBR Green Iaided amplification strategy. Sensors and Actuators B: Chemical, 305, 127348

70. Jarczewska, M., Sheelam, S. R., Ziółkowski, R., \& Górski, Å. (2015). A Label-Free Electrochemical DNA Aptasensor for the Detection of Dopamine. Journal of The Electrochemical Society, 163, B26-B31

71. Wang, Y., Li, Y., Tang, L., Lu, J., \& Li, J. (2009). Application of graphene-modified electrode for selective detection of dopamine. Electrochemistry Communications, 11, 889-892 
72. Wei, B., Zhong, H., Wang, L., Liu, Y., Xu, Y., Zhang, J. ... Wang, H. (2019). Facile preparation of a collagen-graphene oxide composite: A sensitive and robust electrochemical aptasensor for determining dopamine in biological samples. International Journal of Biological Macromolecules, $135,400-406$

73. Liang, Y., Guo, T., Zhou, L., Offenhäusser, A., \& Mayer, D. (2020). Label-Free Split Aptamer Sensor for Femtomolar Detection of Dopamine by Means of Flexible. Organic Electrochemical Transistors, 13, 2577

74. Liu, L., Xia, N., Meng, J. J., Zhou, B. B., \& Li, S. J. (2016). An electrochemical aptasensor for sensitive and selective detection of dopamine based on signal amplification of electrochemical-chemical redox cycling. Journal of Electroanalytical Chemistry, 775, 58-63

75. Zheng, Y., Wang, Y., \& Yang, X. (2011). Aptamer-based colorimetric biosensing of dopamine using unmodified gold nanoparticles. Sensors and Actuators B: Chemical, 156, 95-99

76. Feng, J. J., Guo, H., Li, Y. F., Wang, Y. H., Chen, W. Y., \& Wang, A. J. (2013). Single Molecular Functionalized Gold Nanoparticles for Hydrogen-Bonding Recognition and Colorimetric Detection of Dopamine with High Sensitivity and Selectivity. ACS Applied Materials \& Interfaces, 5, 1226-1231

77. Ren, L., Hang, X., Qin, Z., Zhang, P., Wang, W., Zhang, Y., \& Jiang, L. (2020). Determination of dopamine by a label-free fluorescent aptasensor based on AuNPs and carbon quantum dots. Optik, 208,163560

78. Huang, H., Shi, S., Gao, X., Gao, R., Zhu, Y., Wu, X.... Yao, T. (2016). A universal label-free fluorescent aptasensor based on Ru complex and quantum dots for adenosine, dopamine and $17 \beta$-estradiol detection. Biosensors and Bioelectronics, 79, 198-204

\section{Figures}



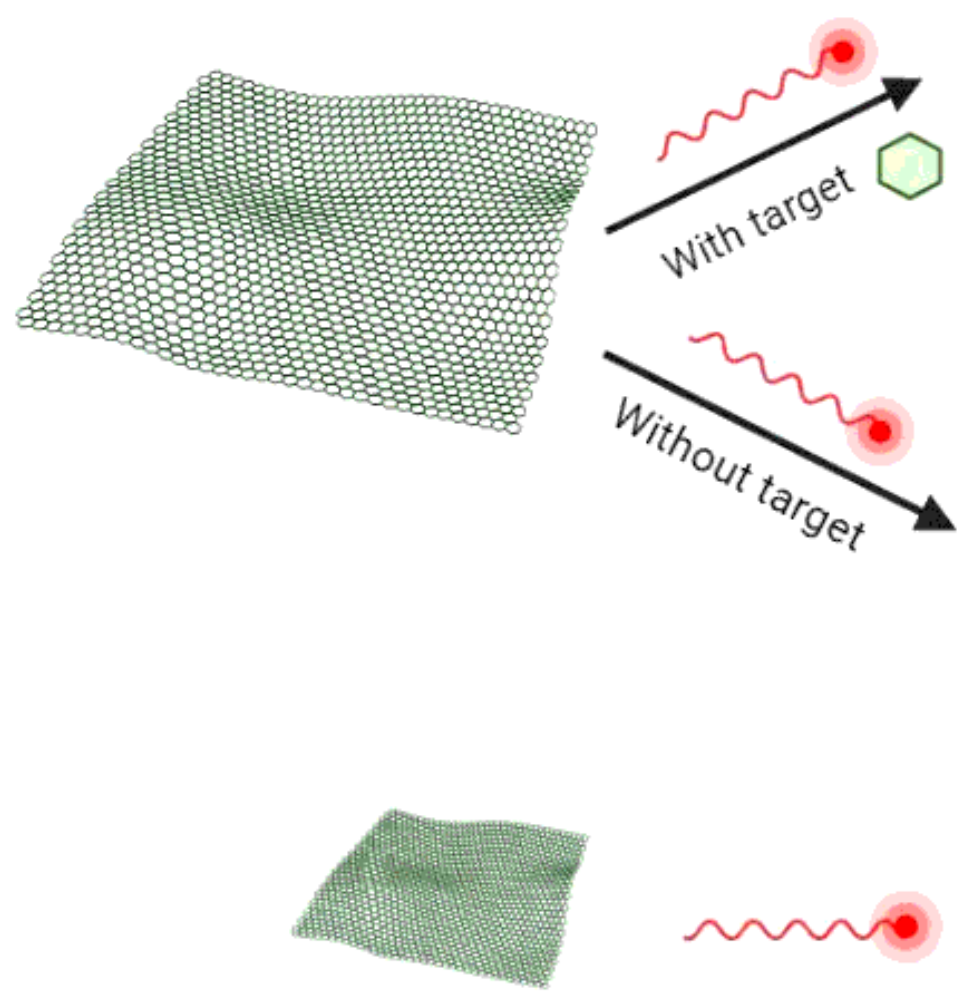

Graphene oxide

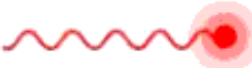

FAM-Aptamer
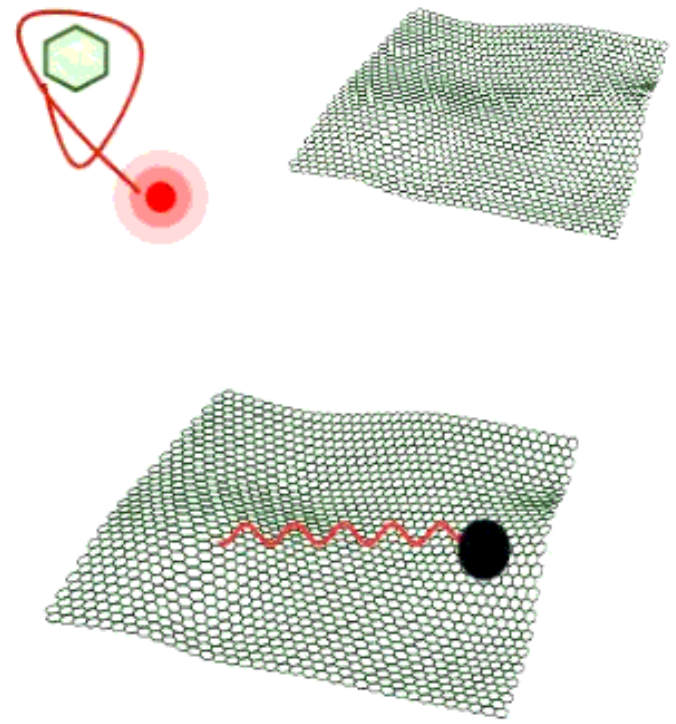

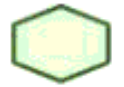

Dopamine

\section{Figure 1}

Schematic illustration for the developed GO-based fluorescent aptasensors for DA detection 


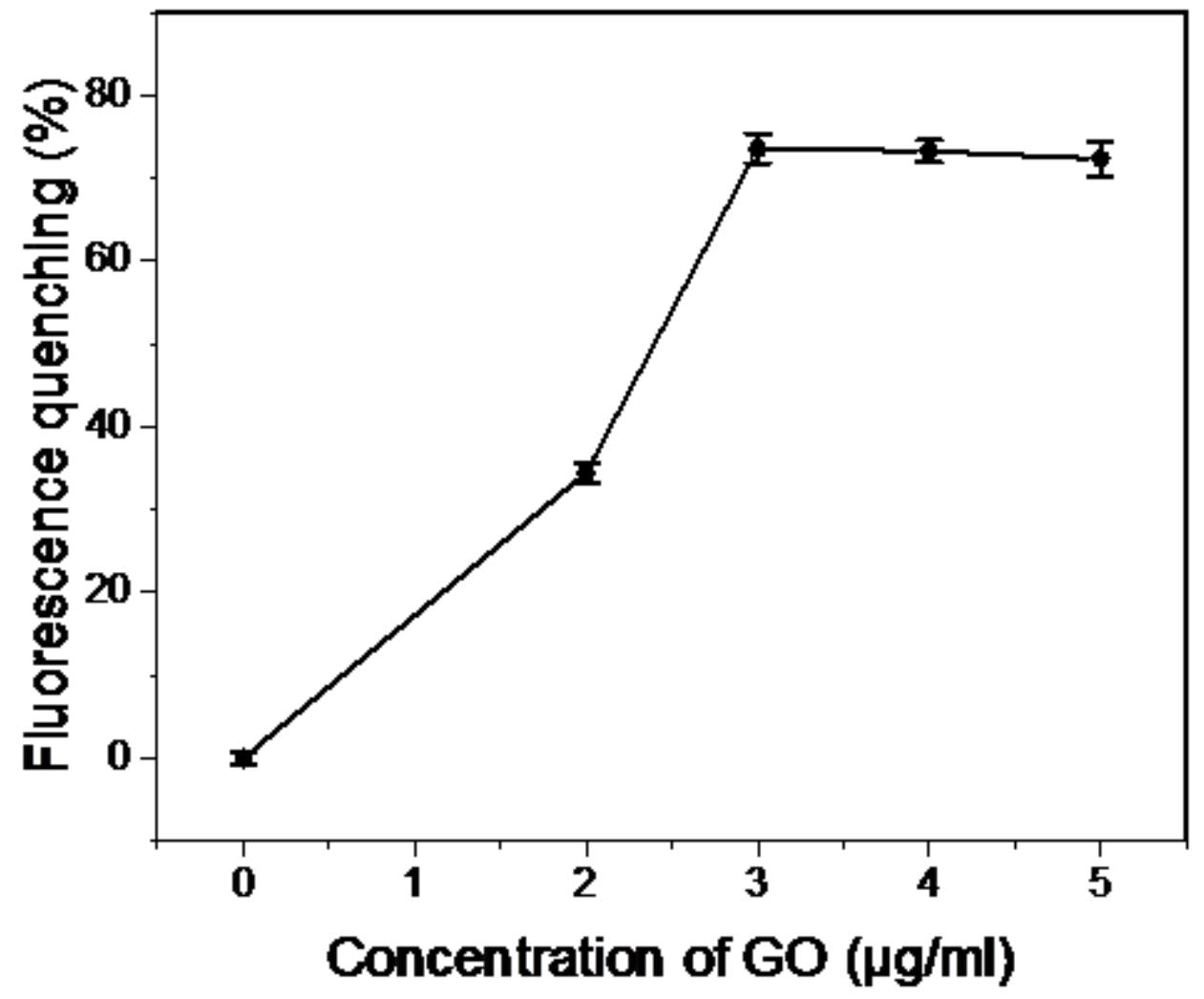

Figure 2

Effect of GO concentration $(0-5 \mu \mathrm{g} / \mathrm{ml})$ on the quenching efficiency toward FAM modified aptamer at the emission wavelength of $538 \mathrm{~nm}$. The aptamer concentration was fixed at $400 \mathrm{nM}$. Error bars were obtained from three experiments 


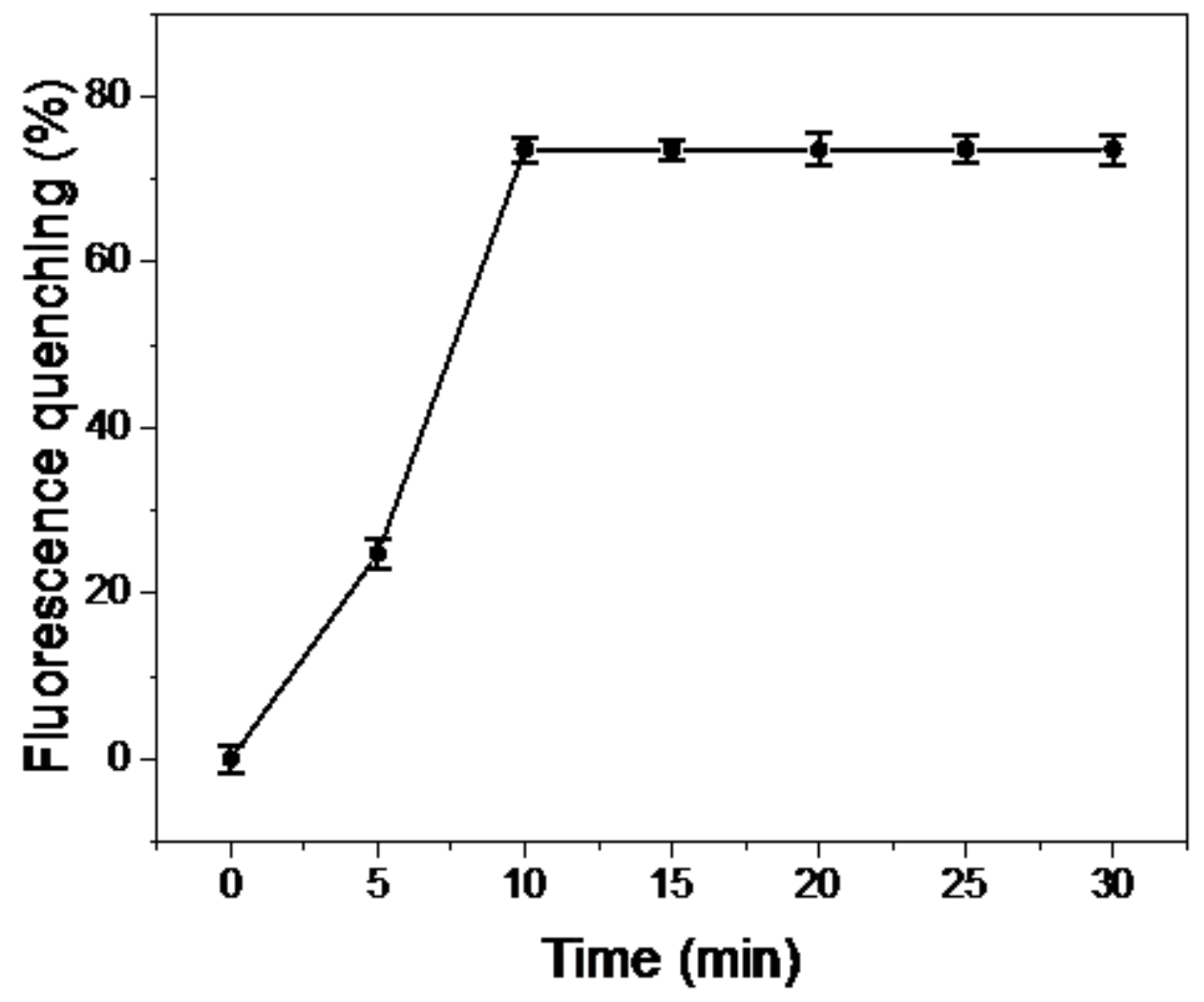

Figure 3

FAM-labeled aptamer quenching kinetic behaviors with respect to the reaction time in the presence of 400 nM FAM-aptamer, and $3 \mu \mathrm{g} / \mathrm{ml} \mathrm{GO}$ in Tris-HCl buffer $(5 \mathrm{mM} \mathrm{MgCl} 2,0.5 \mathrm{M} \mathrm{NaCl}$ and $50 \mathrm{mM}$ Tris HCl, pH 7.4) at the emission wavelength of $538 \mathrm{~nm}$. Error bars were obtained from three experiments 


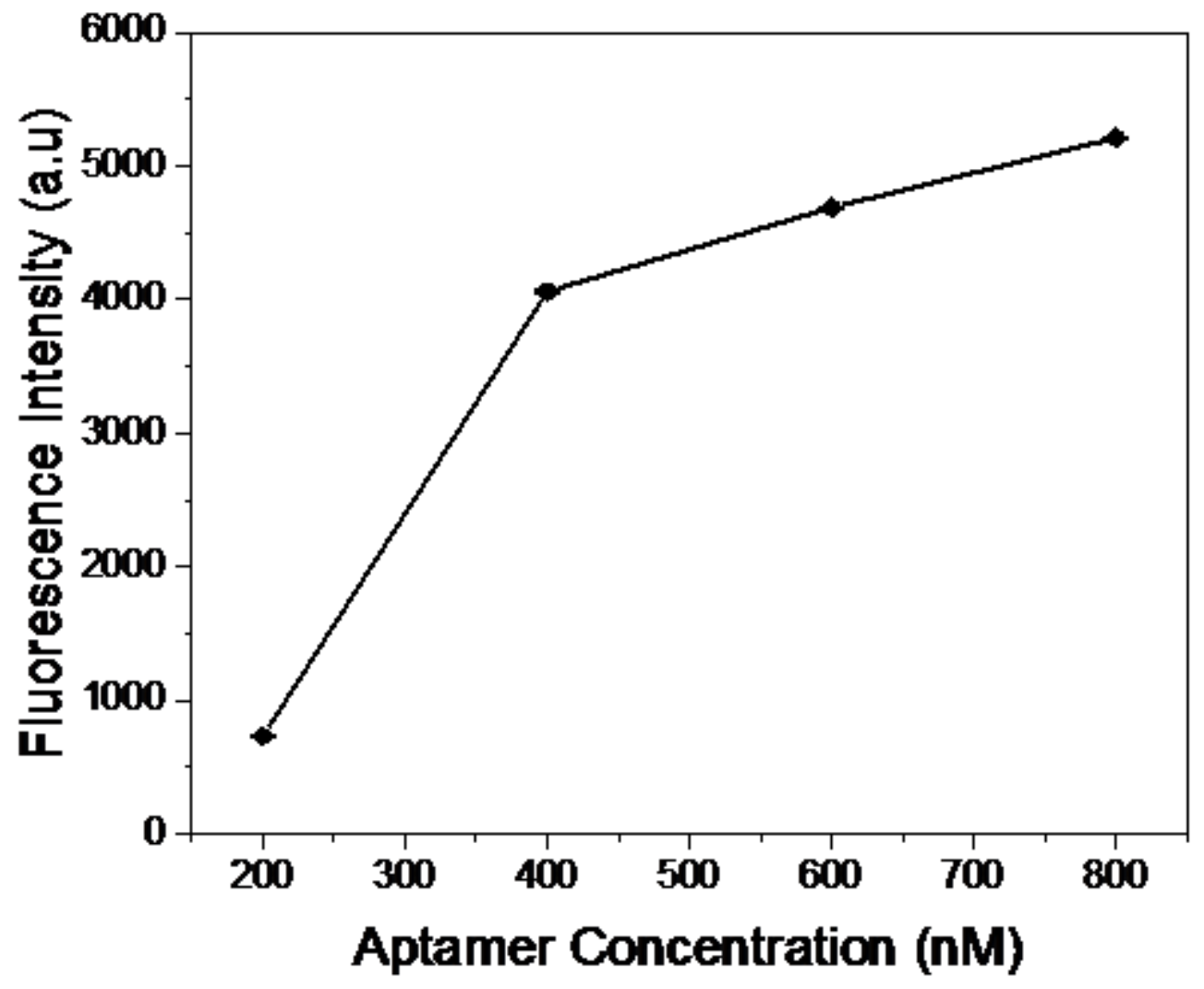

Figure 4

Effect of aptamer concentrations (200-800 nM) on the Fluorescence intensity at the emission wavelength of $538 \mathrm{~nm}$. Error bars were obtained from three experiments 


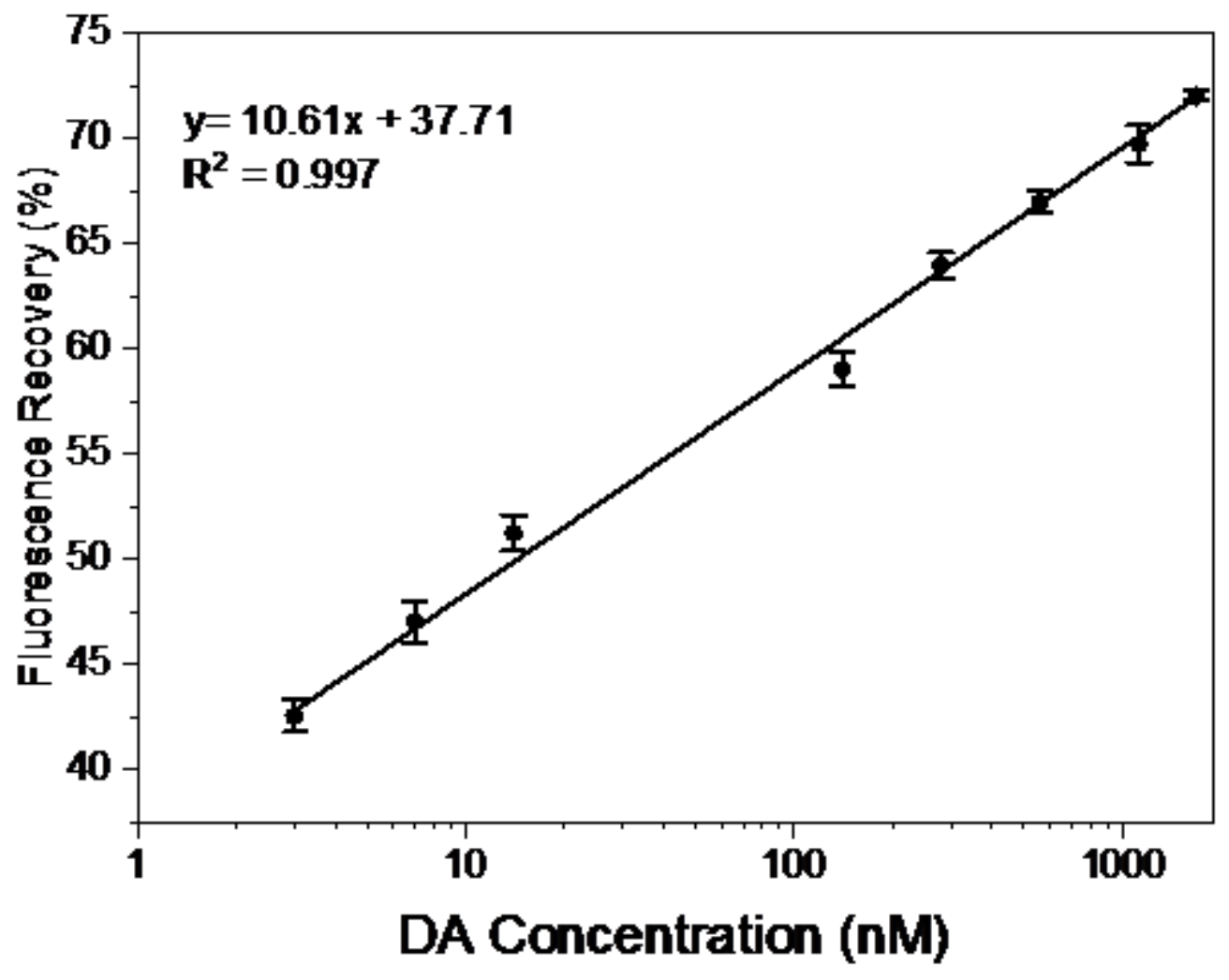

Figure 5

Calibration curve of fluorescence recovery as a function of DA concentrations: $3,7,14,140,280,560$, 1200, $1680 \mathrm{nM}$. Conditions: FAM-aptamer concentration: $400 \mathrm{nM}$. GO concentration: $3 \mu \mathrm{g} / \mathrm{ml}$. Excitation: $485 \mathrm{~nm}$, and emission: $538 \mathrm{~nm}$. Error bars were obtained from three experiments 


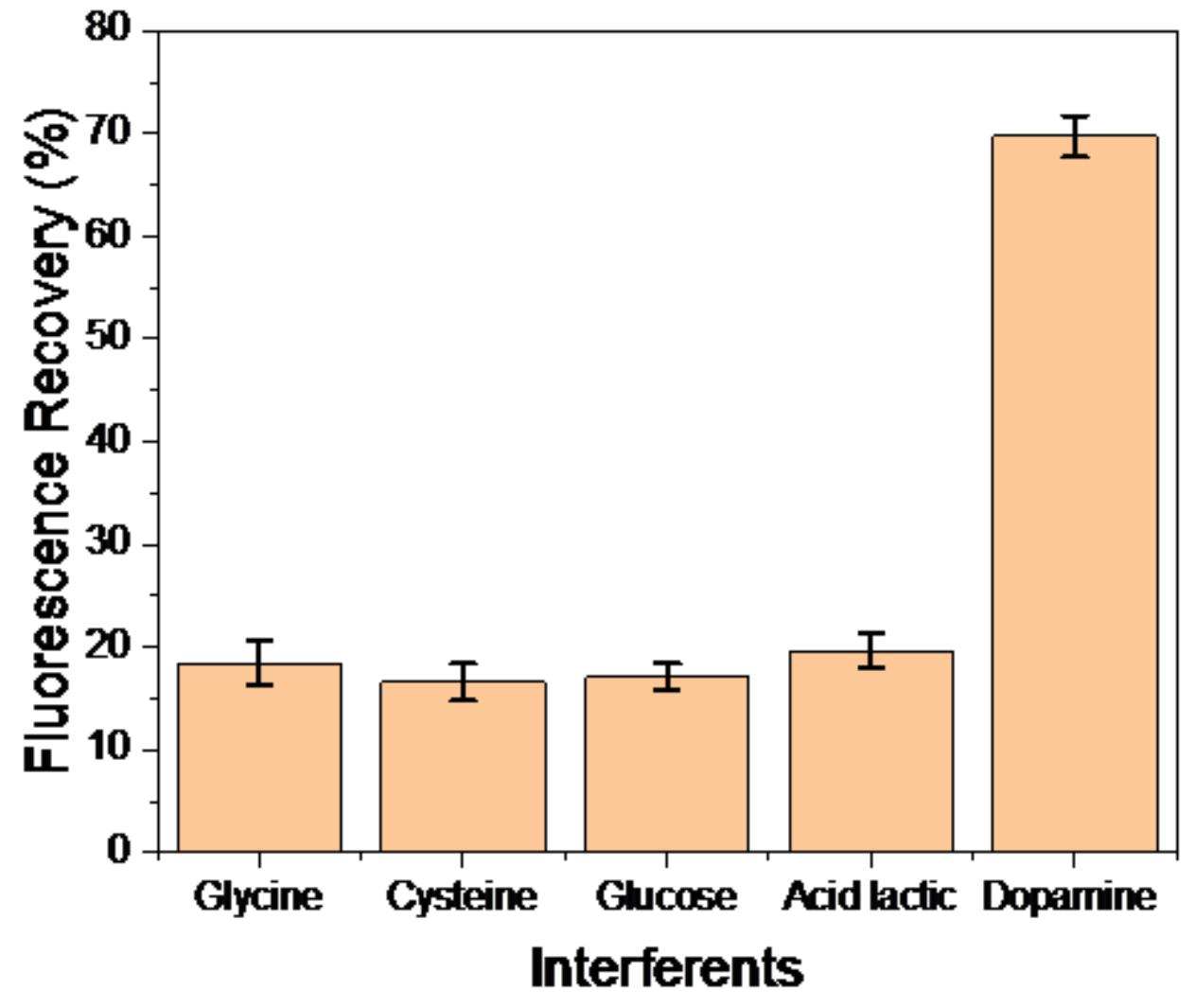

Figure 6

Selectivity of the proposed sensor. The concentrations of all the interferents and DA are $1120 \mathrm{nM}$. Conditions: FAM-aptamer concentration: $400 \mathrm{nM}$. GO concentration: $3 \mu \mathrm{g} / \mathrm{ml}$. Excitation: $485 \mathrm{~nm}$, and emission: $538 \mathrm{~nm}$. Error bars were obtained from three experiments 


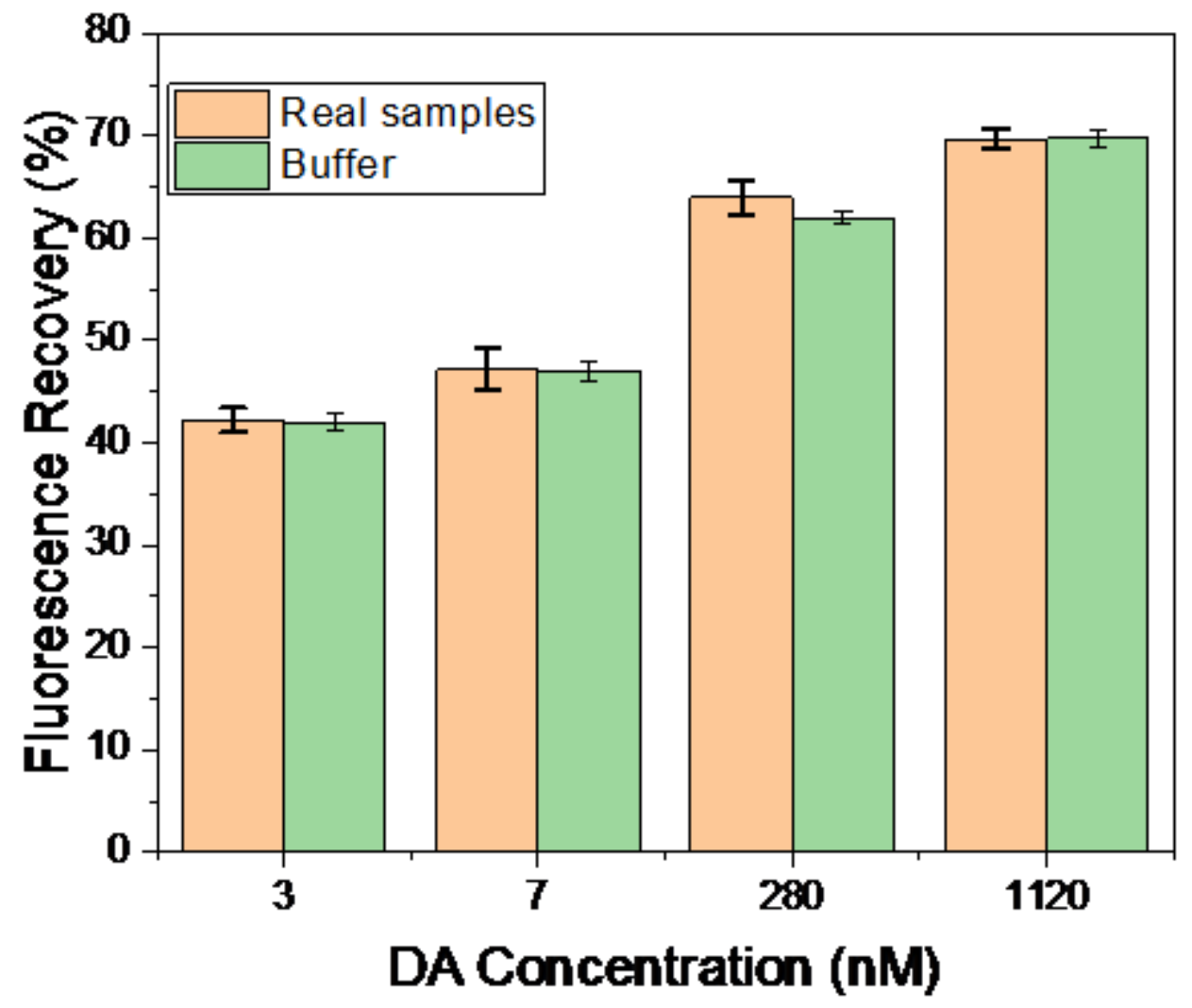

Figure 7

Comparison of fluorescence recoveries efficiency between buffer (green bars) and human serum samples (orange bars). Conditions: FAM-aptamer concentration: $400 \mathrm{nM}$. GO concentration: $3 \mu \mathrm{g} / \mathrm{ml}$. Excitation: $485 \mathrm{~nm}$, and emission: $538 \mathrm{~nm}$. Error bars were obtained from three experiments 